INPE-9880-PRE/5455

IMPROVING THE RATE MONOTONIC AND THE FIRST

DEADLINE FIRST SCHEDULERS FOR REAL TIME

SIMULATION AND CONTROL OF AEROSPACE VEHICLES

Gilberto da Cunha Trivelato*

Marcelo Lopes de Oliveira e Souza

*EMBRAER- Empresa Brasileira de Aeronáutica

Paper presented at the AIAA Modeling and Simulation Technologies Conference, 11-14 Aug 2003, Austin, Texas, USA. 


\title{
IMPROVING THE RATE MONOTONIC AND THE FIRST DEADLINE FIRST SCHEDULERS FOR REAL TIME SIMULATION AND CONTROL OF AEROSPACE VEHICLES
}

\author{
Gilberto da Cunha Trivelato, M.Sc. \\ EMBRAER - Empresa Brasileira de Aeronáutica S. A. \\ P.O. Box 8050 \\ 12227-901 - S. José dos Campos - SP - Brasil \\ trivelato@embraer.com.br \\ Marcelo Lopes de Oliveira e Souza, Ph.D. \\ INPE - National Institute for Space Research \\ DMC - Division of Space Mechanics and Control \\ Av. dos Astronautas, 1758 \\ 12227-010 - S. José dos Campos - SP - Brasil \\ marcelo@dem.inpe.br
}

\begin{abstract}
In this work we discuss and improve the rate monotonic scheduler and the first deadline first scheduler for real time simulation and control. These algorithms are largely know and discussed in the real time literature but in such literature: 1) some aspects of their original propositions are/remain incomplete or hidden; 2) there are even some counter-examples and critiques to them made in recent works. Correspondingly, in this work: 1) we discuss and clarify some aspects of their original propositions and we improve such propositions by explicitly writing down some of their incomplete or even hidden passages and transformations; 2) we also discuss and clarify some mistakes done in those counter-examples and critiques, by replacing them with new examples that confirm the algorithms and illustrate the points discussed, clarified, and improved. We also suggest some possible extensions of those algorithms and their uses in real time simulation and control of aerospace vehicles, especially aircrafts and satellites.
\end{abstract}

KEYWORDS: Scheduling Techniques, Rate Monotonic Algorithm, First Deadline First Algorithm, Real Time Simulation, Real Time Control, Aerospace Vehicles

\section{INTRODUCTION}

Even today with incredible and powerful computers, experience shows that we are always looking for more processing time for new and more complex tasks. There are some special embedded applications where we cannot replace and/or upgrade computers anytime but the environment always changes: an operation flight program for a fighter in a complex an dynamic defense system, a spaceship or spacecraft in an interplanetary trip flying through debris, or a future microcomputer inside human body as a complementary part of its defense or behavior systems. These systems need to be "hard real time".

"Hard real time" system is a system where the service must be guaranteed for each task before the next request for the same task. A short and good bibliography revision about real time systems is done by Farines et all $^{1}$. Then the scheduling program ("scheduler") in a single processor is always studied

Copyright (C) 2003 by G. C. Trivelato and M. L. O. Souza

Published by The American Institute of Aeronautics

and Astronautics, Inc., with permission. from the viewpoint of its most important constraint: guaranteed service. In general we consider a parallel processor machine as a "single" processor machine with finite processing resources. Fujimoto ${ }^{2}$ presents a detailed study about parallel and distributed simulation. A Schedulers are the rules that determine the time for the set of tasks to be executed. There are many schedulers in the literature including the Rate Monotonic Scheduler (RMS) and the First Deadline First Scheduler (FDFS) or Earliest Deadline First Scheduler (EDS).

In this work we discuss and improve the rate monotonic scheduler and the first deadline first scheduler for real time simulation and control. These algorithms are largely know and discussed in the real time literature but in such literature: 1) some aspects of their original propositions are/remain incomplete or hidden; 2) there are even some counter-examples and critiques to them made in recent works. Correspondingly, in this work: 
1) we discuss and clarify some aspects of their original propositions and we improve such propositions by explicitly writing down some of their incomplete or even hidden passages and transformations; 2) we also discuss and clarify some mistakes done in those counter-examples and critiques, by replacing them with new examples that confirm the algorithms and illustrate the points discussed, clarified, and improved. We also suggest some possible extensions of those algorithms and their uses in real time simulation and control of aerospace vehicles, especially aircrafts and satellites.

\section{ENVIRONMENT}

The hard-real time environment has the same assumptions presented in Liu and Layland ${ }^{3}$ as follows:

"(A1) The requests for all tasks for which hard deadlines exist are periodic, with constant intervals between requests.

(A2) Deadlines consist of run-ability constraints only i.e. each task must be completed before the next request for it occurs.

(A3) The tasks are independent in that requests for a certain task do not depend on the initiation or the completion of requests for other tasks.

(A4) Run-time for each task is constant for that task and does not vary with time. Run-time here refers to the time that is taken by a processor to execute the task without interruption.

(A5) Any non-periodic tasks in the system are special; they are initialization or failure-recovery routines; they displace periodic tasks while they themselves are being run, and do not themselves have hard, critical deadlines."

As a consequence, we can have a complete characterization of a task by its request period $\left(T_{i}\right)$ and their run time $\left(C_{i}\right)$. Then, in the same way as defined by Liu and Layland" "in this paper we shall use $\tau_{1}$, $\tau_{2}, \ldots, \tau_{m}$ to denote $m$ periodic tasks with their request periods being $T_{1}, T_{2}, \ldots, T_{m}$ and their runtimes $C_{1}, C_{2}, \ldots, C_{m}$, respectively. The request rate of a task is defined to be the reciprocal of its request period."

\section{A FIXED PRIORITY SCHEDULING ALGORITHM}

The basic definitions are the same presented in Liu and Layland ${ }^{3}$. A static scheduler assigns a priority to each task in the design time and it remains the same. But a dynamic scheduler assigns such priority in run-time, so it is variable. The Rate Monotonic Scheduler (RMS) is static and it assigns a fixed priority to each process based on its period: the shorter the period the higher the priority. But a First Deadline First Scheduler (FDFS) or Earliest Deadline First (EDF) is a dynamic scheduler that has explicit information about deadlines and assigns higher priority to the process with the nearest deadline. A preemptive scheduler immediately suspends the current task and starts a higher priority task whenever it is requested. "The deadline of a request for a task is defined to be the time of the next request for the same task." In a scheduling algorithm, "we say that an overflow occurs at time $t$ if $t$ is the deadline of an unfulfilled request". "A critical instant for a task is defined to be an instant at which a request for that task will have the largest response time". The next critical instant will happen in the minimum common multiple (MCM) for all tasks. "A critical time zone for a task is the time interval between a critical instant and the end of the response to the corresponding request of the task". A set of tasks "fully utilize the processor if the priority assignment is feasible for the set and if an increase in the run-time of any of the tasks in the set will make the priority assignment infeasible". The task skew is the time delay between the beginning of $T_{i}$ and $C_{i}$.

Liu and Layland ${ }^{3}$ proposed theorem 1: A critical instant for any task occurs whenever the task is requested simultaneously with requests for all higher priority tasks.

Proof: Liu and Layland ${ }^{3}$. The proof is clear and easy to understand. The result in theorem 1 also suggests a priority assignment that is optimum in the sense that will be stated in theorem 2. "Such an assignment of priorities will be known as the rate monotonic priority assignment. As it turns out, such a priority assignment is optimum in the sense that no other fixed priority assignment rule can schedule a task set that cannot be scheduled by the rate-monotonic priority assignment." "If the requests for all tasks at their critical instants are fulfilled before their respective deadlines, then the scheduling algorithm is feasible."

Liu and Layland ${ }^{3}$ proposed theorem 2: If a feasible priority assignment exists for some task set, the rate monotonic priority assignment is feasible for that task.

Proof: See Liu and Layland ${ }^{3}$. The proof is clear and easy to understand, but we clarify that it is only applied to the fixed schedulers family. 


\section{PROCESSOR UTILIZATION FACTOR}

Liu and Layland ${ }^{3}$ defined processor utilization factor to be the fraction of processor time spent in the execution of the task set. Since $\left(C_{i} / T_{i}\right)$ is the fraction of processor time spent in executing task $\tau_{i}$, for $m$ tasks, the utilization factor is:

$$
U\left(C_{i}, T_{i}\right)=\sum_{i=1}^{m}\left(C_{i} / T_{i}\right) .
$$

However, in this definition, we should clarify, that (1) can be rewritten as follows:

$$
U\left(C_{i}, T_{i}, M\right)=\sum_{i=1}^{m}\left\{\left(M / T_{i}\right) .\left(C_{i} / M\right)\right\}
$$

where $M$ is the minimum common multiple (MCM) among all task periods. If they are mutually prime, then $M=T_{1} \cdot T_{2} \ldots T_{m}$. Defining $n_{i}=M / T_{i}$ as a number of times that task $i$ appears in the interval time $M$, we get:

$$
U\left(n_{i}, C_{i}, M\right)=\left\{\sum_{i=1}^{m}\left(n_{i} \cdot C_{i}\right)\right\} / M .
$$

We can say that the processor utilization factor is the percentage of time spent by processor executing all tasks in any interval time $M$. If there is no common factor between any $\left(T_{i}, T_{j}\right)$ then, when $m$ grows, $M$ grows faster. Even if we could define a set of tasks with all skews equal to zero that fully utilize the processor considering only the interval $T_{m}$, the utilization factor has real meaning only in the time interval $M$.

Liu and Layland ${ }^{3}$ proposed theorem 3: For a set of two tasks with fixed priority assignments, the least upper bound (lub) to the processor utilization factor is $U_{\text {lub }}=2 \cdot\left(2^{1 / 2}-1\right)$.
We clarify the proof including the graphical representation of the task set in both cases.

Let $\tau_{1}, \tau_{2}$ be two tasks with periods being $T_{1}$ and $T_{2}$ and run-times being $C_{1}$ and $C_{2}$, respectively. Assume that $T_{1}>T_{2}$ w.o.l.o.g. According to the rate-monotonic priority assignment $\tau_{1}$ has higher priority than $\tau_{2}$. In a critical time zone of $\tau_{2}$, there are $\left\lceil T_{2} / T_{1}\right\rceil$ ("ceiling" of $T_{2} / T_{1}=$ smallest integer $\geq T_{2} / T_{1}$ ) requests for $\tau_{1}$. If we adjust $C_{2}$ to fully utilize the processor we have two cases:

Case 1: all requests of $\tau_{1}$ within the critical time zone of $T_{2}$ are completed before the second $\tau_{2}$ request Figure 1.

Using the results from Liu and Layland ${ }^{3}$, where $C_{2}=C_{2}\left(C_{1}, T_{1}, T_{2}\right)$, we have:

$$
U\left(C_{1}, T_{1}, T_{2}\right)=1+C_{1} \cdot \underbrace{\left[\left(1 / T_{1}\right)-\left(1 / T_{2}\right),\left[T_{2} / T_{1}\right]\right]}_{\leq 0}
$$

In this case, the processor utilization factor $U\left(C_{1}, T_{1}, T_{2}\right)$ is monotonically decreasing with $C_{1}$.

Case 2: the execution of $\left\lceil T_{2} / T_{1}\right\rceil^{\text {th }}$ request for $\tau_{1}$ overlaps the second $\tau_{2}$ request - Figure 2 .

Using the results from Liu and Layland ${ }^{3}$, where $C_{2}=C_{2}\left(C_{1}, T_{1}, T_{2}\right)$ and $\left\lfloor T_{2} / T_{1}\right\rfloor$ is "floor" of $T_{2} / T_{1}=$ greatest integer $\leq T_{2} / T_{1}$, we have:

$$
U=\left(T_{1} / T_{2}\right)\left\lfloor T_{2} / T_{1}\right\rfloor+C_{1} \cdot \underbrace{\left(1 / T_{1}\right)-\left(1 / T_{2}\right)\left\lfloor T_{2} / T_{1}\right\rfloor}_{\geq 0} .
$$

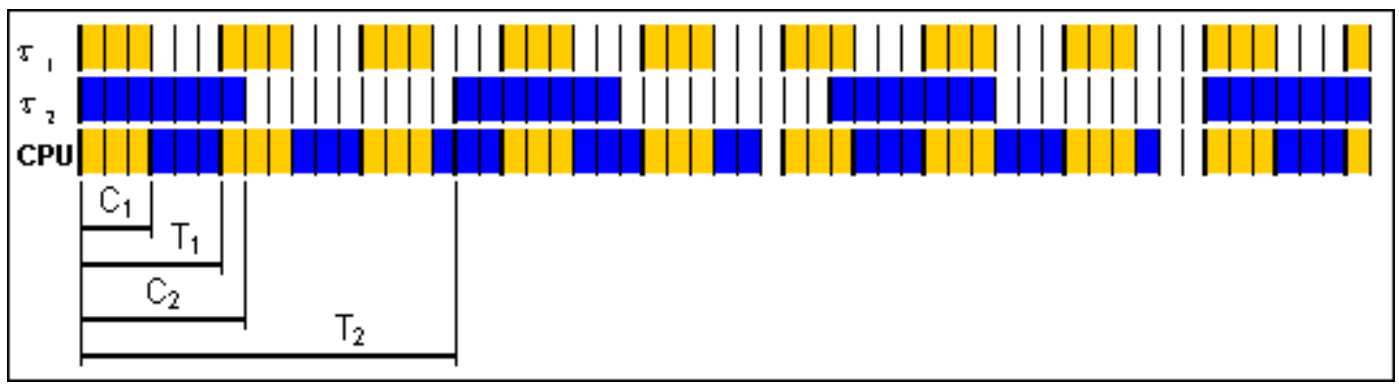

Figure 1 - Task scheduling for case 1, with $\tau_{1}=\left\{T_{1}=6, C_{1}=3\right\}, \tau_{2}=\left\{T_{2}=16, C_{2}=7\right\}$ time units. 


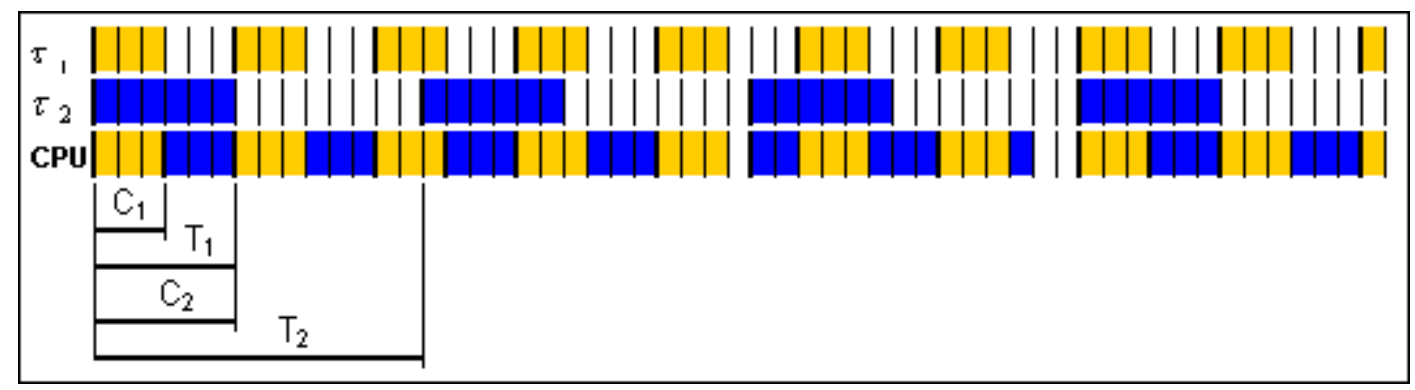

Figure 2 - Task scheduling for case 2, with $\tau_{1}=\left\{T_{1}=6, C_{1}=3\right\}, \tau_{2}=\left\{T_{2}=14, C_{2}=6\right\}$ time units.

In this case, the processor utilization factor $U\left(C_{1}, T_{1}, T_{2}\right)$ is monotonically increasing with $C_{1}$.

The minimum of $U\left(C_{1}, T_{1}, T_{2}\right)$ clearly occurs at the boundary between (3) and (4). Then:

$$
\left.\left.C_{1}=T_{2}-T_{1}\right\rfloor T_{2} / T_{1}\right\rfloor
$$

As shown in Liu and Layland ${ }^{3}$ this value is:

$\left.\left.U\left(T_{1}, T_{2}\right)=1-\left(T_{1} / T_{2}\right) \cdot \llbracket T_{2} / T_{1}\right\rceil-\left(T_{2} / T_{1}\right)\right] .\left[\left(T_{2} / T_{1}\right)-\left\lfloor T_{2} / T_{1}\right\rfloor\right.$

and the least upper bound of $U\left(T_{1}, T_{2}\right)$ for all $T_{1}, T_{2}$ is numerically $U_{\text {lub }}=2 .\left(2^{1 / 2}-1\right) \approx 0.83$.

Other Liu and Layland ${ }^{3}$ proposition is theorem 4: For $a$ set of $m$ tasks with fixed priority order, and the restriction that the ratio between any two request periods is less than 2, the least upper bound to the processor utilization factor is $U_{\text {lub }}=m \cdot\left(2^{1 / m}-1\right)$.

Proof: Let $\tau_{1}, \tau_{2}, \ldots, \tau_{m}$ to denote $m$ periodic tasks. Let $C_{1}, C_{2}, \ldots, C_{m}$ be the run-times of the tasks that fully utilize the processor and minimize the processor utilization factor. Assume that $T_{1}<T_{2}<\ldots<T_{m-1}<T_{m}$. Let $U\left(C_{i}, T_{i}\right)$ denote the processor utilization factor.

Lets proof the theorem as Liu and Layland ${ }^{3}$ and illustrate it with $\tau_{1}=\left\{T_{1}=8, C_{1}^{\text {lub }}=3\right\}$, $\tau_{2}=\left\{T_{2}=11, C_{2}^{\text {lub }}=4\right\}$ and $\tau_{3}=\left\{T_{3}=15, C_{3}^{\text {lub }}=1\right\}$.

We wish to show that (lemma 1): $C_{1}^{\text {lub }}=T_{2}-T_{1}$.

Suppose that $C_{1}=T_{2}-T_{1}+\Delta, \Delta>0, \Delta=1$ then, we have a new task $\tau_{1}=\left\{T_{1}=8, C_{1}=4\right\}$, $\tau_{2}=\left\{T_{2}=11, C_{2}=3\right\}$ and $\tau_{3}=\left\{T_{3}=15, C_{3}=1\right\}$ as shown in Figure 3 and clearly fully utilize the processor. It is equivalent to transfer $\Delta$ from task $\tau_{2}$ to task $\tau_{1}$. The first $\Delta$ from $C_{2}^{\text {lub }}$ compensates directly $C_{1}$. The second $\Delta$, added to second $C_{1}$, postpone the second $C_{2}$ by $\Delta$ with no overflow because it is the same amount left from the end.

Lets rewrite the task as $C_{1}^{\prime}, C_{2}^{\prime}, \ldots, C_{m}^{\prime}$, where:

$$
\begin{aligned}
& C_{1}^{\prime}=T_{2}-T_{1} \\
& C_{2}^{\prime}=C_{2}+\Delta \\
& C_{3}^{\prime}=C_{3} \\
& \vdots \\
& C_{m-1}^{\prime}=C_{m-1} \\
& C_{m}^{\prime}=C_{m}
\end{aligned}
$$

If $U^{\prime}$ denotes the corresponding utilization factor, we have:

$$
U-U^{\prime}=\left(\Delta / T_{1}\right)-\left(\Delta / T_{2}\right)>0 .
$$

Some important facts that are not in Liu and Layland ${ }^{3}$, but are introduced here, are that: if $\tau_{i}, i=2, \ldots m$, satisfies $C_{i}>\Delta$, we can transfer $\Delta$ from any task $\tau_{i}(1<i \leq m)$ to $\tau_{1}$ and the result is still valid, i.e., $U_{\text {lub }}-U^{i}=\left(\Delta / T_{1}\right)-\left(\Delta / T_{i}\right)>0$. And more, $U_{\text {lub }}-U^{i}<U_{\text {lub }}-U^{i+1}$ because $T_{i}<T_{i+1}$.

Alternatively, suppose that $C_{1}=T_{2}-T_{1}-\Delta$, $\Delta>0, \Delta=1$ then, we have a new task $\tau_{1}=\left\{T_{1}=8, C_{1}=2\right\}, \tau_{2}=\left\{T_{2}=11, C_{2}=6\right\}$ and $\tau_{3}=\left\{T_{3}=15, C_{3}=1\right\}$ as shown in Figure 4 and clearly fully utilize the processor. It is equivalent to transfer $\Delta$ from task $\tau_{1}$ to task $\tau_{2}$. Because, under 


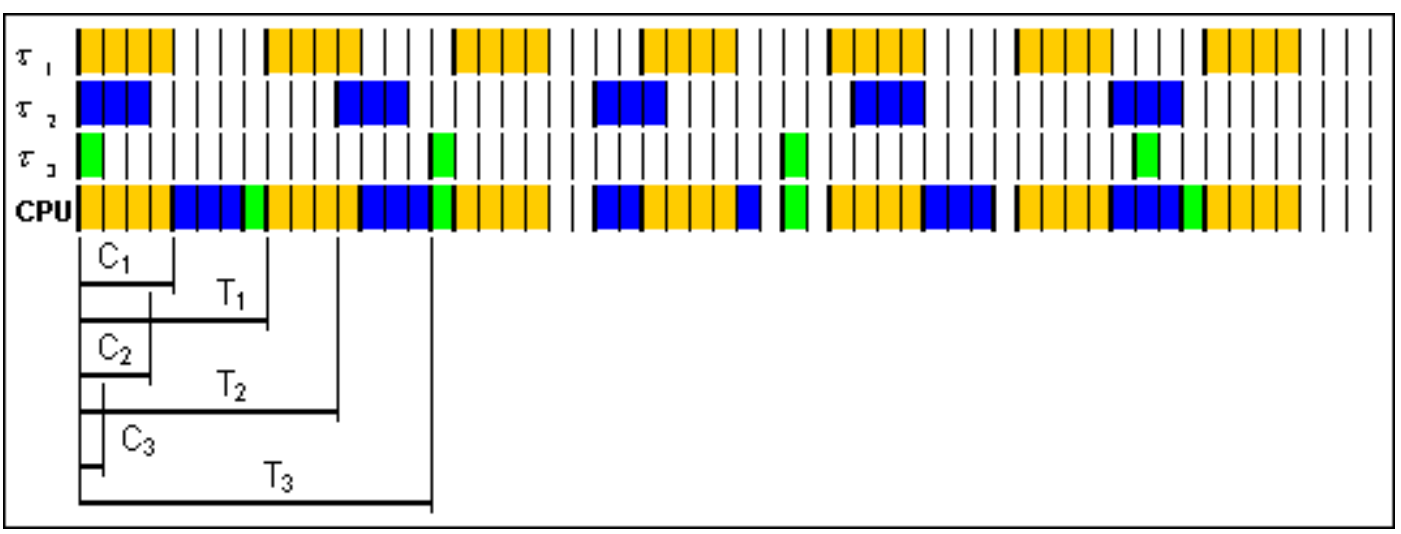

Figure 3 - Full utilization with $C_{1}=T_{2}-T_{1}+\Delta$.

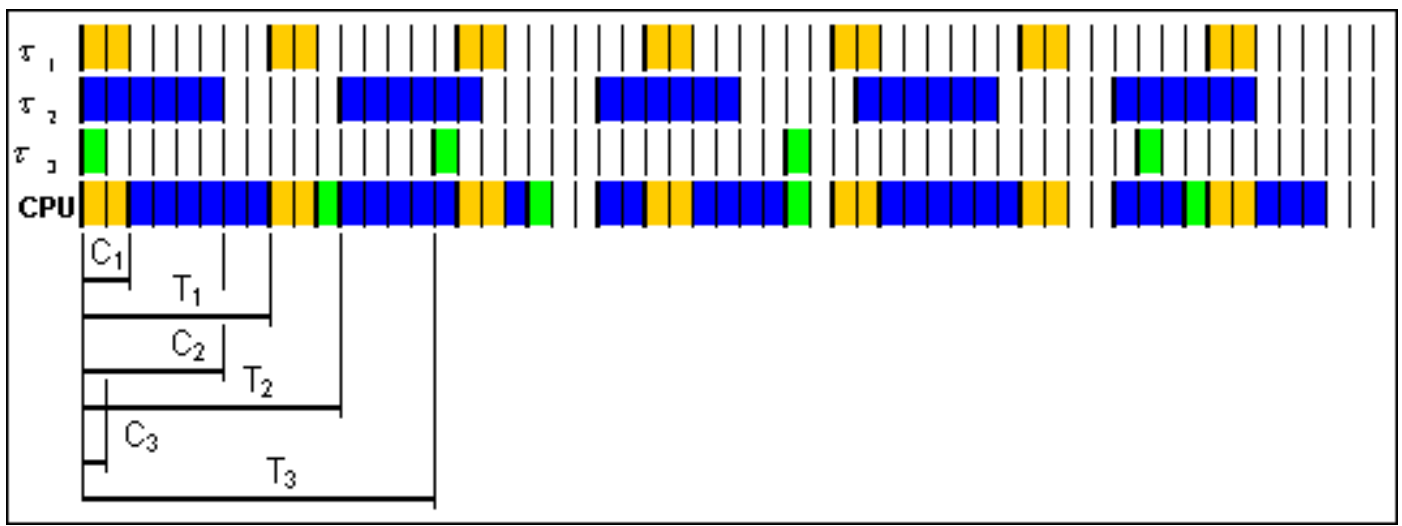

Figure 4 - Full utilization with $C_{1}=T_{2}-T_{1}-\Delta$.

theorem hypothesis, there are always two $C_{1}^{\text {lub }}$ executions before the second $\tau_{2}$ request in time interval $T_{2}$, we must add in $C_{2}$ twice the $\Delta$ value subtracted from $C_{1}^{\text {lub }}$. In Figure 4 we can verify that in the first time interval $T_{2}$ we have 2 times $C_{1}$ executions, in the first interval $T_{3}$ we also have 2 times $C_{2}$ executions, and first $C_{3}$ is always executed just before a completion of first time interval $T_{1}$.

Lets rewrite the task as $C_{1}^{\prime \prime}, C_{2}^{\prime \prime}, \ldots, C_{m}^{\prime \prime}$, where:

$$
\begin{aligned}
& C_{1}^{\prime \prime}=T_{2}-T_{1} \\
& C_{2}^{\prime \prime}=C_{2}-2 . \Delta \\
& C_{3}^{\prime \prime}=C_{3}
\end{aligned}
$$

$$
\begin{aligned}
& C_{m-1}^{\prime \prime}=C_{m-1} \\
& C_{m}^{\prime \prime}=C_{m}
\end{aligned}
$$

If $U^{\prime \prime}$ denotes the corresponding utilization factor, we have:

$$
U-U^{\prime \prime}=-\left(\Delta / T_{1}\right)+2 .\left(\Delta / T_{2}\right)>0 .
$$

Some important facts that are not in Liu and Layland ${ }^{3}$, but are introduced here, are that we can we can cut $\Delta$ from task $\tau_{1}$ and add $2 . \Delta$ to any $\tau_{i}(1<i \leq m)$ and the result is still valid, i.e., $U_{\text {lub }}-U^{i}=-\left(\Delta / T_{1}\right)+\left(2 . \Delta / T_{i}\right)>0$, and again, $U_{\text {lub }}-U^{i}<U_{\text {lub }}-U^{i+1}$. 
Therefore, if indeed $U$ is the minimum of full utilization factors, then $C_{1}=T_{2}-T_{1}$. In the previous numerical example we can verify that two modified task sets $C_{1}^{\prime}, C_{2}^{\prime}, \ldots, C_{m}^{\prime}$ and $C_{1}^{\prime \prime}, C_{2}^{\prime \prime}, \ldots, C_{m}^{\prime \prime}$ satisfy this condition and are equal to $C_{1}^{\text {lub }}, C_{2}^{\text {lub }}, \ldots, C_{m}^{\text {lub }}$.

Now we wish to show that (lemma 2): $C_{m}^{\text {lub }}=T_{m}-T_{m-1}$.

Liu and Layland ${ }^{3}$ did say, "in a similar way we can prove that", as shown in Liu and Layland ${ }^{3}$ :

$$
\begin{aligned}
& C_{2}=T_{3}-T_{2} \\
& C_{3}=T_{4}-T_{3} \\
& \quad \vdots \\
& C_{m-1}=T_{m}-T_{m-1} \\
& C_{m}=T_{m}-2 .\left(C_{1}+C_{2}+\ldots+C_{m-1}\right)
\end{aligned}
$$

But they did not prove it in Liu and Layland ${ }^{3}$. So, lets clarify this using Figure 5.

The rest of the proof is as in Liu and Layland ${ }^{3}$; and it gives as the important result:

$$
U_{\operatorname{lub}(m)}^{m}=m \cdot\left(2^{1 / m}-1\right) .
$$

Devillers and Goossens ${ }^{4}$ used a numerical example $\tau_{1}=\left\{T_{1}=8, C_{1}=2\right\}, \tau_{2}=\left\{T_{2}=11, C_{2}=3\right\}$ and $\tau_{3}=\left\{T_{3}=15, C_{3}=5\right\}$ as a "counter example" to show some "mistakes" in theorem 4. Lets clarify the theorem 4 and the "counter example" to have better understanding of both. Consider the task $\tau_{1}=\left\{T_{1}=8, C_{1}\right\}, \tau_{2}=\left\{T_{2}=11, C_{2}\right\} \quad$ and $\tau_{3}=\left\{T_{3}=15, C_{3}\right\}$, with the same $T_{1}, T_{2}, T_{3}$ used in the "counter example" of Devillers and Goossens ${ }^{4}$ and all feasible $C_{1}, C_{2}, C_{3}$ combinations. The processor utilization factor for all feasible $C_{1}, C_{2}, C_{3}$ combinations are presented in Table 1 and Figure 6. The $C_{1}$, values are in rows, the $C_{2}$ values are in columns, and the $C_{3}$ values are calculated to fully utilize the processor.

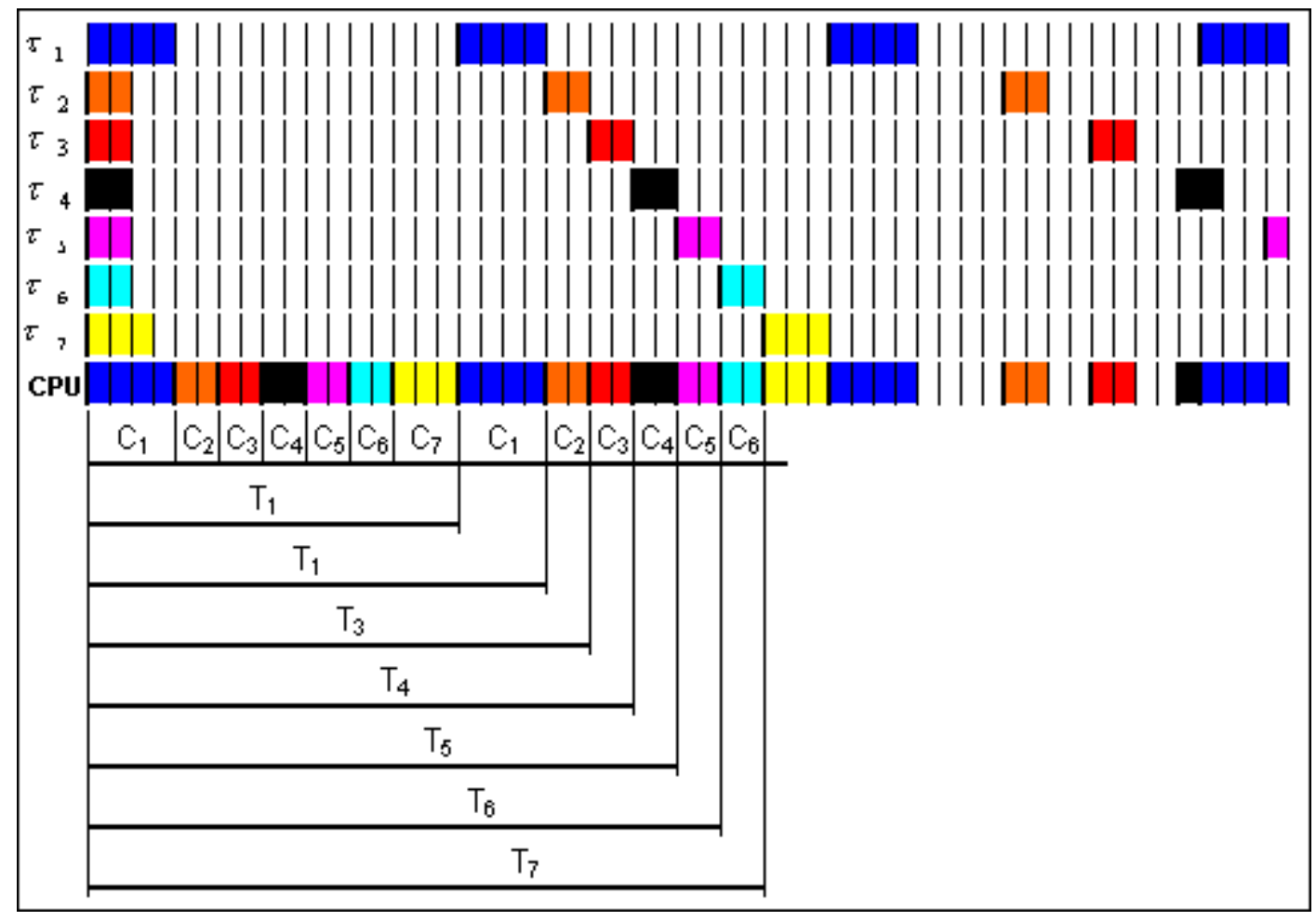

Figure 5 - Full utilization for a task set with $\mathbf{m}=7$. 
Table 1 - Full processor utilization factor for all feasible $C_{1}, C_{2}, C_{3}$ combinations.

\begin{tabular}{|c|c|c|c|c|c|c|}
\hline $\mathbf{C}_{\mathbf{2}} \backslash \mathbf{C}_{\mathbf{1}}$ & 1 & 2 & 3 & 4 & 5 & 6 \\
\hline 1 & 1,083 & 0,941 & 0,933 & 0,924 & 0,916 & 0,908 \\
\hline 2 & 0,907 & 0,898 & 0,890 & 0,882 & 0,873 & - \\
\hline 3 & 0,864 & 0,856 & 0,848 & 0,839 & - & - \\
\hline 4 & 0,822 & 0,814 & $\mathbf{0 , 8 0 5}$ & - & - & - \\
\hline 5 & 0,846 & 0,838 & - & - & - & - \\
\hline 6 & 0,870 & 0,862 & - & - & - & - \\
\hline 7 & 0,895 & - & - & - & - & - \\
\hline 8 & 0,919 & - & - & - & - & - \\
\hline
\end{tabular}

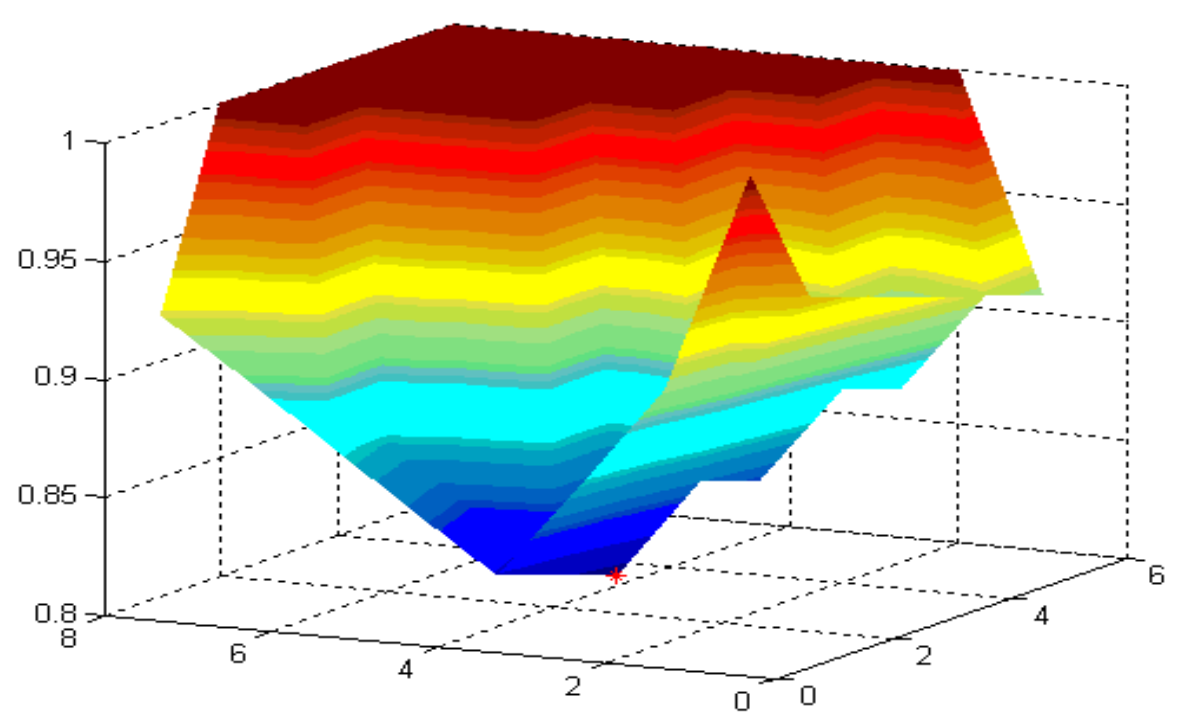

Figure 6 - Utilization factor $U\left(C_{1}, C_{2}\right)$ with $C_{3}$ chosen to fully utilize the processor.

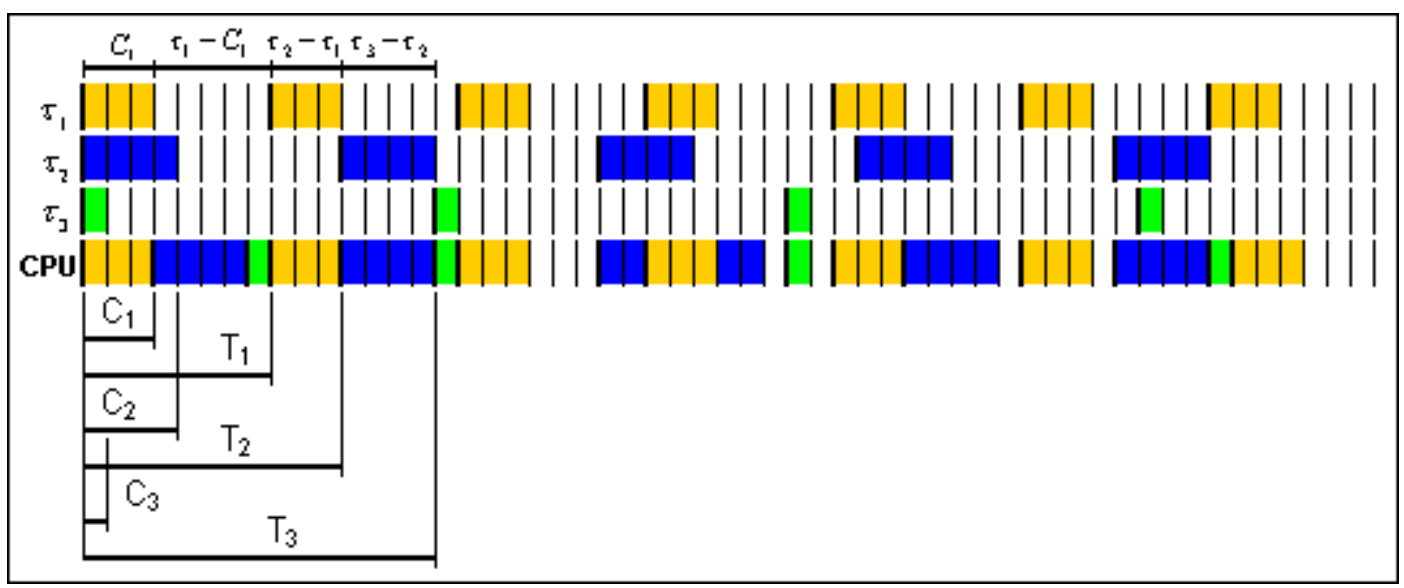

Figure 7 - Task set for minimum $\mathrm{U}$ and fully utilization. 
The minimum $U$ is $U_{\text {lub }}=0.805$ at task $\tau_{1}, \tau_{2}, \tau_{3}$, $\tau_{1}=\left\{T_{1}=8, C_{1}^{\text {lub }}=3\right\}, \quad \tau_{2}=\left\{T_{2}=11, C_{2}^{\text {lub }}=4\right\}$ and $\tau_{3}=\left\{T_{3}=15, C_{3}^{\text {lub }}=1\right\}$. This task set is presented in Figure 7.

So the "counter example" of Devillers and Goossens ${ }^{4}$ is wrong because in order to disprove theorem 4 such "counter example" must satisfy the hypothesis of theorem 4. But we cannot choose the combination $\tau_{1}=\left\{T_{1}=8, C_{1}=2\right\}, \quad \tau_{2}=\left\{T_{2}=11, C_{2}=3\right\}$ and $\tau_{3}=\left\{T_{3}=15, C_{3}=5\right\}$ used in Devillers and Goossens ${ }^{4}$ because it fully utilizes the processor and provides $U=0.856$, but it does not minimize the processor utilization factor. Theorem 4 says that we must use the task $\left\{\tau_{1}=\left\{T_{1}=8, C_{1}^{\text {ub }}=3\right\}\right.$, $\left.\tau_{2}=\left\{T_{2}=11 C_{2}^{\text {dub }}=4\right\} \quad \tau_{3}=\left\{T_{3}=15, C_{3}^{\text {lub }}=1\right\}\right\}$ that minimizes the processor utilization factor among all $C_{1}, C_{2}, C_{3}$ combinations that satisfies full utilization only.

Liu and Layland ${ }^{3}$ generalized the theorem 4 as theorem 5: For a set of $m$ tasks with fixed priority order, the least upper bound to the processor utilization factor is $U_{\operatorname{lub}(m)}^{m}=m \cdot\left(2^{1 / m}-1\right)$.

Proof: to do that they proved that for any task set that fully utilize the processor, the processor utilization factor $U^{\prime}$ is less than or equal $U_{\text {lub }}^{m}\left(U^{\prime} \leq U_{\text {lub }}^{m}\right)$ and, consequently, to determine the least upper bound of the processor utilization factor we need only consider task sets in which the ratio between any two request periods is less than two.

\section{MISUNDERSTANDINGS}

In the specific literature, as in Burns and Wellings ${ }^{5}$, page 347, we see simpler forms of Liu and Layland ${ }^{3}$ equations and conclusions for schedulability: "for a single processor system, a set of $m$ independent, periodic processes with only execution-time deadlines is schedulable if the overall processor utilization is less than $m .\left(2^{1 / m}-1\right)$. For a large $m$ this asymptotically approaches 0.693 . Therefore, all processes sets are schedulable if processor utilization is less than 69\%". (M1) Looking quickly, at first impression, we can wrongly think that if the processor utilization factor is greater than $m \cdot\left(2^{1 / m}-1\right)$ (for $m$ finite) or than $69,3 \%$ $(m \rightarrow \infty)$ the task set is not feasible using RMS. This result assumes that all task periods $T_{1} \cdot T_{2} \ldots T_{m}$ are mutually prime, the worst possible relationship among them. If there is more favorable relationship among them the system can achieve higher utilization factor. In some special cases, as one shown in Burns and Wellings $^{5}$, p. 347, and reproduced in Figure 8, $\tau_{1}=\left\{T_{1}=80, C_{1}=40\right\}, \tau_{2}=\left\{T_{2}=40, C_{2}=10\right\}$ and $\tau_{3}=\left\{T_{3}=20, C_{3}=5\right\}, U$ reaches $100 \%$.

(M2) On the other hand, we can wrongly think, if using RMS and the processor utilization time is less than 69,3 $\%(m \rightarrow \infty)$ we have available time to add tasks to the processor because for large $m$ the processor utilization factor asymptotically approaches 0.693 . We already saw it as a specification item in a simulation contract. More than that, we faced a real problem where there was available time in the processor and we could add nothing. In fact, for a task that fully utilizes the processor if at least one time unit is added, it will be infeasible even there is available processor time. In this situation we need evaluate a new scheduler or, in worst case, replace the processor with a faster one.

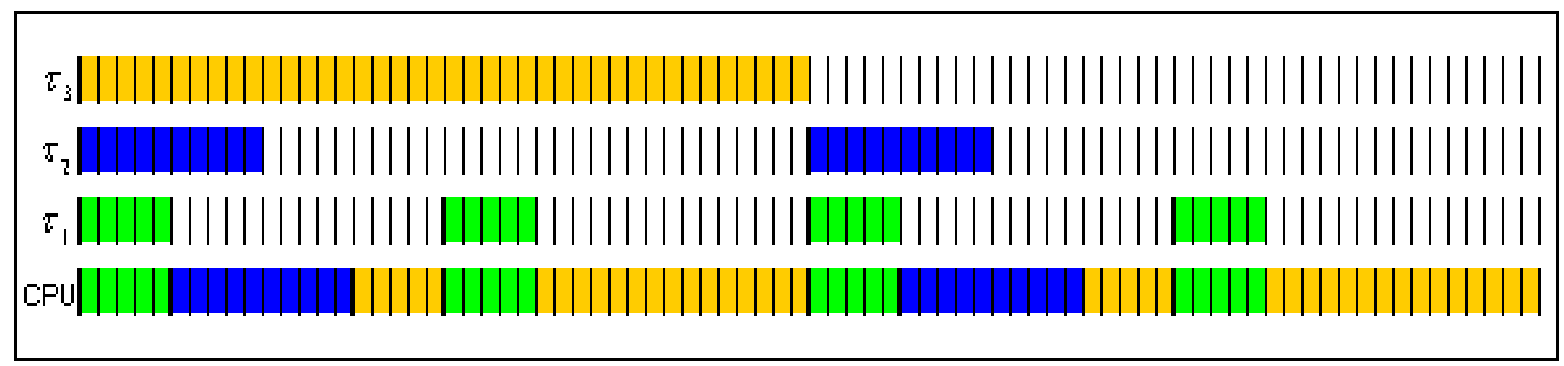

Figure 8 - Task set for $\tau_{1}=\left\{T_{1}=80, C_{1}=40\right\}, \tau_{2}=\left\{T_{2}=40, C_{2}=10\right\}$ and $\tau_{3}=\left\{T_{3}=20, C_{3}=5\right\}$. 


\section{RATE MONOTONIC SCHEDULER (RMS) RESTRICTIONS}

In the previous sections we demonstrated that using RMS all task sets are schedulable if the processor utilization factor is less than $69,3 \%$ using worst possible relationship (mutually prime) among all task periods. We can improve it choosing $T_{1} \cdot T_{2} \ldots T_{m}$ with good relationships among $\left(T_{i}, T_{j}\right)$. As shown in the previous example, the simplest way to do achieve $100 \%$ processor utilization factor using RMS is to make null the fractional part of $T_{m} / T_{i},\left\{T_{m} / T_{i}\right\}=0$, for $i=1,2, \ldots, m-1$. It is good, but not enough, because in the real world this can no be always done and because it still necessary to consider the switching costs between tasks.

\section{FIRST DEADLINE FIRST SCHEDULER (FDFS)} OR EARLIEST DEADLINE FIRST (EDF)

In the second part of the paper Liu and Layland ${ }^{3}$ proved that FDFS or EDF scheduler can schedule any tasks that are schedulable by any other method; and the least upper bound on the processor utilization factor is uniformly $100 \%$. Liu and Layland ${ }^{3}$ proposed and proved the theorem 7 (their number): for a given set of $m$ tasks, the deadline driven scheduling algorithm is feasible if and only if

$$
\left(C_{1} / T_{1}\right)+\left(C_{2} / T_{2}\right)+\ldots+\left(C_{m} / T_{m}\right) \leq 1 .
$$

A FDFS graphical representation for the task $\tau_{1}=\left\{T_{1}=8, C_{1}=3\right\}, \tau_{2}=\left\{T_{2}=11, C_{2}=4\right\}$ and $\tau_{3}=\left\{T_{3}=15, C_{3}=1\right\}$ used in theorem 4 is presented in Figure 9.

True, it improves the RMS; but in real world it is a complex implementation and it does not solve the problem of switching costs between tasks.

\section{IMPROVEMENTS ON THE RMS AND FDF SCHEDULERS}

Even though the Rate Monotonic Scheduler and the First Deadline First Scheduler are based in solid theory in scheduling area, they are not suitable for a complex and dynamic simulation environment with great number of players (big number of tasks) mostly because they do not solve the problem of switching costs among tasks and can guarantee the service only for a known number of tasks. To overcome these problems it is necessary and possible to do some extensions of those algorithms in order to apply them to an environment that always changes like: an operation flight program for a fighter in a complex an

dynamic defense system; a spaceship or spacecraft in an interplanetary trip flying through debris; or a future microcomputer inside human body as a complementary part of its defense or behavior systems. This will be presented and detailed in a future paper.

\section{CONCLUSIONS}

In this work we discussed and clarified that the rate monotonic scheduler and the first deadline first scheduler are solid theory for real time simulation and control. These algorithms are largely know and discussed in the real time literature; but in this work: 1) we discussed and clarified some aspects of their original propositions and we improved such

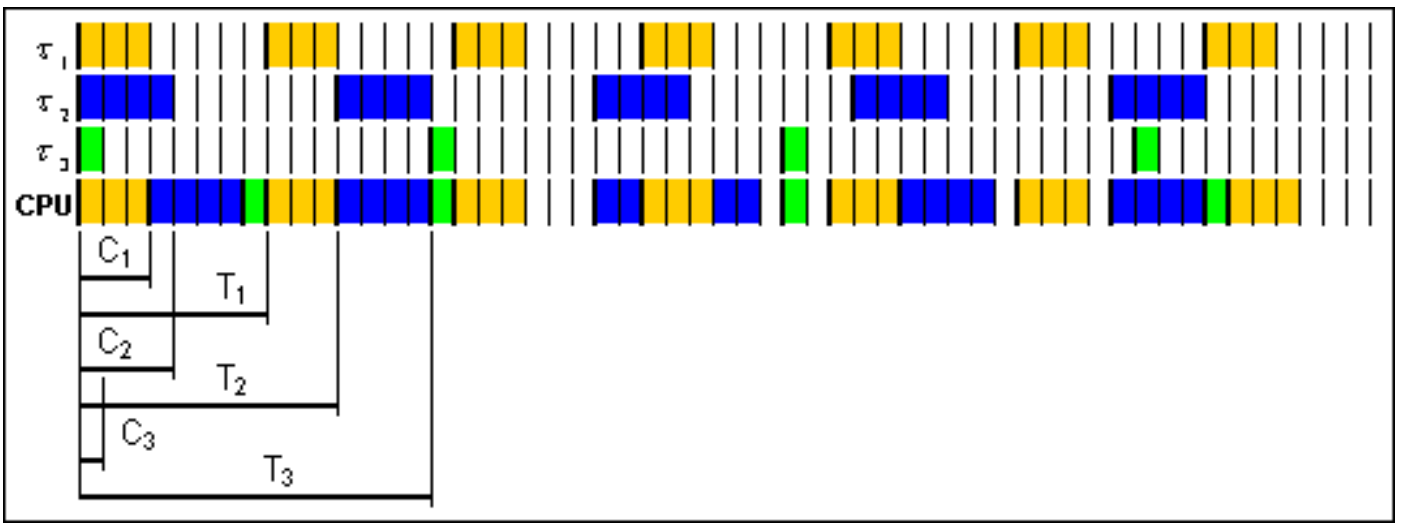

Figure 9- FDFS for task $\tau_{1}=\left\{T_{1}=8, C_{1}=3\right\}, \tau_{2}=\left\{T_{2}=11, C_{2}=4\right\}$ and $\tau_{3}=\left\{T_{3}=15, C_{3}=1\right\}$. 
propositions by explicitly writing down some of their incomplete or even hidden passages and transformations; 2) we also discussed and clarified some mistakes done in those counter-examples and critiques, by replacing them with new examples that confirm the algorithms and illustrate the points discussed and clarified.

\section{REFERENCES}

[1] Farines, J.M., Fraga, J.S., Oliveira, R.S. "Sistemas de Tempo Real", IME-USP, São Paulo, Brasil, 2000, 201 pp.
[2] Fujimoto, R. M. "Parallel and Distributed Simulation Systems", John Wiley \& Sons, NY, EUA, 2000, $300 \mathrm{pp}$.

[3] Liu, C. L. and Layland, J.W., 1973, "Scheduling Algorithms for Multiprogramming in Hard-realtime environment", J. ACM 20 (1), 16 pp.

[4] Devillers, R. and Goossens, J., 2000, "Liu and Layland's Schedulability Test Revisited", Information Processing Letters 73, 5 pp.

[5] Burns, A.; Wellings, A. "Real-time Systems and Their Programming Languages", Addison-Wesley, Reading, MA, USA, 1990, 575p. 